\title{
Intelligence Competition by Sudoku Game with Java Software (ICSGJS)
}

\author{
Abdulmonem I. Shennat
}

\begin{abstract}
This paper aims to match the fun and simplicity of the game of Sudoku Game at the same time as adding some new features. It will include a timer clock, with a single player feature that will calculate their time and recode it in SQLITE data base with the full name of the player, thus setting up a challenge to human players. It then allows the next player to start a new game and repeats the same process until the last player, at which point the time spent by each player can be calculated and the competition draws to an exciting close. This paper explores a new design in the traditional Sudoku Game to make it more interesting and challenging. The simplicity of this game makes it an ideal candidate for a minor project as we can focus on advanced topics like Intelligence Competition Functionality and Sudoku Game with Java Software (ICFSGJS).
\end{abstract}

Index Terms-Intelligence competition, multiplayer game, package of main classes, unique feature.

\section{INTRODUCTION}

\section{A. Background Information on Sudoku}

There have been various puzzles throughout history that were Sudoku-like, the creator of the modern version has been credited to Howard Garns, an architect from Indianapolis. He created them for Dell Magazine in 1979 and called them "Number Place." Eventually, these puzzles made their way to a Japanese magazine in 1984. The magazine renamed these puzzles as Sudoku, which meant "single numbers". It didn't take long for Sudoku to become popular in Japan. But it was only when a British newspaper called "The Times" decided to publish the puzzle in 2004 that Sudoku became a worldwide phenomenon. Today, Sudoku puzzles are published in dozens of magazines in over 35 countries. There are also numerous books and computer games dedicated to this puzzle [1].

\section{B. Traditional Sudoku Game}

The Sudoku game is a logic-based, number-placement puzzle that is a special form of Latin Squares. It is most often a puzzle played on a $9 \times 9$ grid that can only contain numbers. "Fig. 1," shows a typical Sudoku puzzle. The player is normally required to fill each cell of the board with numbers one to nine, following specified rules. The rules are normally that each number cannot appear more than once on a row, on a column, and on a block. A block is normally defined as a 3 $\times 3$ subset of the board, and there are nine blocks in the entire

Manuscript received January 10, 2014; revised March 18, 2014.

Abdulmonem I. Shennat is with the Faculty of Information Technology in Misuratauniversity, Misurata 051, Libya (e-mail: abdulmonem_ibrahim@hotmail.com). grid. "Fig. 2," shows the same puzzle in "Fig. 1" with solution numbers marked in red. Although this form is the most popular version of Sudoku, there are many other variations, ranging from a standard Sudoku grid but with additional rules to unusual board arrangements like jigsaws and geometric shapes other than squares [2].

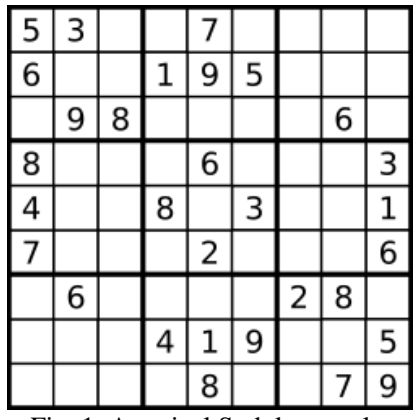

Fig. 1. A typical Sudoku puzzle.

\begin{tabular}{|l|l|l|l|l|l|l|l|l|}
\hline 5 & 3 & 4 & 6 & 7 & 8 & 9 & 1 & 2 \\
\hline 6 & 7 & 2 & 1 & 9 & 5 & 3 & 4 & 8 \\
\hline 1 & 9 & 8 & 3 & 4 & 2 & 5 & 6 & 7 \\
\hline 8 & 5 & 9 & 7 & 6 & 1 & 4 & 2 & 3 \\
\hline 4 & 2 & 6 & 8 & 5 & 3 & 7 & 9 & 1 \\
\hline 7 & 1 & 3 & 9 & 2 & 4 & 8 & 5 & 6 \\
\hline 9 & 6 & 1 & 5 & 3 & 7 & 2 & 8 & 4 \\
\hline 2 & 8 & 7 & 4 & 1 & 9 & 6 & 3 & 5 \\
\hline 3 & 4 & 5 & 2 & 8 & 6 & 1 & 7 & 9 \\
\hline
\end{tabular}

Fig. 2. The same puzzle in Fig. 1 with solution numbers marked in red.

\section{Project Background}

Intelligence Competition by Sudoku Game with Java Software (ICSGJS). It is a remake of traditional Sudoku game by java software and recode it in data base for each player with calculated time by the administrator or computer.

This project aims to bring the fun and simplicity of Sudoku game with some new feature. It will include timer clock whose aim will be to challenge the human players. It will also have the multiplayer feature that will allow more than one players to play the game over a network in the same competition. The traditional Sudoku game does not offer much challenge to it's players. Hence players loose interest in the game after playing it for sometime. Offering some sort of challenge and adventure to the players will help increase the addictive power of this game. The multiplayer with timer clock features of this game will make this game more challenging and interesting. The simplicity of this game makes it an ideal candidate for a minor project as we can focus on advanced topics like Intelligence Competition Functionality and Sudoku Game with Java Software (ICFSGJS) [1], [3]. 
The strong support for networked application in Java programming language with data base and availability of high speed network connection layer in present day computers will allow us to build a very efficient multiplayer version of Sudoku game [1].

\section{Problem StATEMENT}

The traditional Sudoku game does not offer much challenge to it's players. It is impossible to bring out the best playing skill of a players unless a challenge is offered to them. The players loose interest in the game after playing it for sometime due to lack of challenge. Hence when people hear about Sudoku game, they quickly form an image of old age arcade game [1], [2].

\section{OBJECTIVES}

This game aims to change the way people think of traditional Sudoku game. It will offer the experience of commercial multiplayer games to the player retaining the simplicity of traditional Sudoku game. The major objectives of this project are:

1) Create a Sudoku game that will have all the functionality of traditional Sudoku games.

2) Introduce multiplayer functionality in the game that will allow several players to play a game one by one in the same match. It should give the experience of real time games to the players.

3) Introduce a new Sudoku (unique feature of this game) to make the game more challenging and interesting. The movement and action of this a new game will be controlled by computer whose aim will be to calculate the total time for each player in one match [3].

\section{PRoposed System}

The proposed system explains the traditional Sudoku game with new features to make this game more interesting with multiplayer game by analysis and design the system. Then, calculating the spent time for each player as a result.

\section{A. System's Algorithm}

"Fig. 3," shows the flow chart of the processes of this new game as an algorithm of this system (ICSGJS) that it explains us how a new competition and challenge between players one by one are done [4].

\section{B. System's Analysis and Design}

To analysis this system we needed to know the system activities that they represent use cases. So, a use case is an activity the system carries out, usually in response to a request by a user of the system. It is a situation in which the system must accomplish some goal of a user and documented in a use case diagram. "Fig. 4," shows use case diagram with five use cases and two users or actors. In this instance, both actors the player and administrator are allowed to access the system directly. As indicated by relationship lines, each actor can use every use case that is related them. In addition to one use case from five use cases "stop the game" is accessed for both the player and administrator [5].

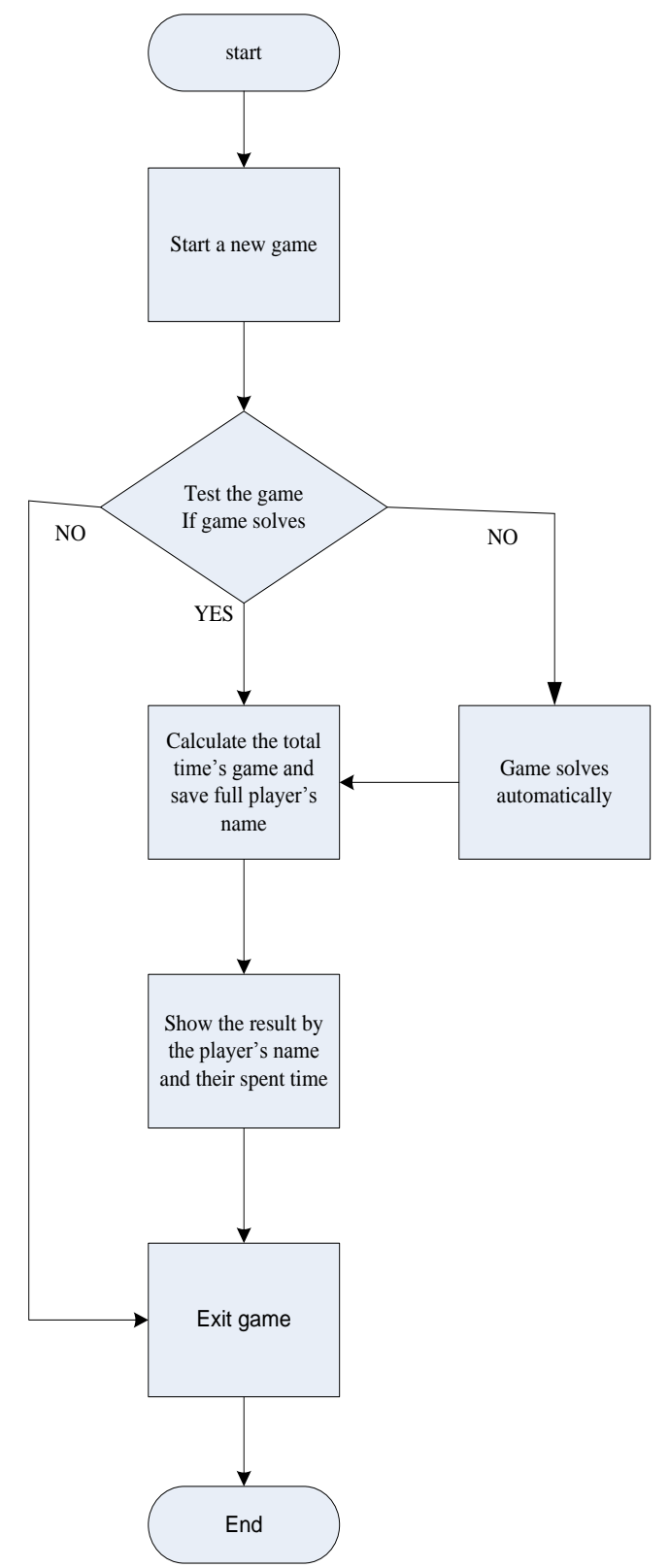

Fig. 3. Flow chart of processes of (ICSGJS) system.

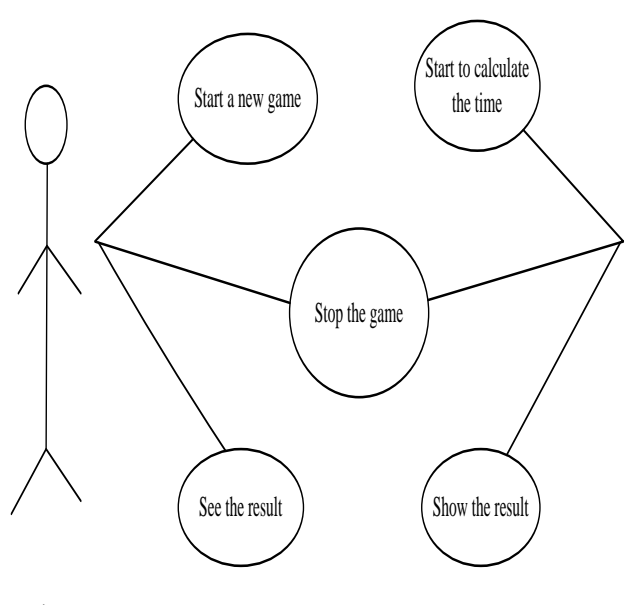

player

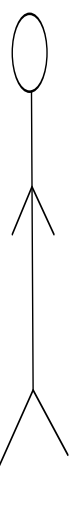

Administrator

Fig. 4. Use case diagram of (ICSGJS) system.

To design this system we used Java program with SQLITE database to get the results. In firefox we could get extension 
SQLITE manager so there we could create database and tables. Here is a package of main classes that were imported. This package is shown below as a sample program of (ICSGJS) system [6], [7].

For applications of this system:

$$
\begin{aligned}
& \text { import java.awt.*; } \\
& \text { importjava.awt.event.*; } \\
& \text { importjava.sql.Connection; } \\
& \text { importjava.sql.Date; } \\
& \text { importjava.sql.PreparedStatement; } \\
& \text { importjava.sql.ResultSet; } \\
& \text { importjava.text.DateFormat; } \\
& \text { importjava.text.SimpleDateFormat; } \\
& \text { importjavax.swing.*; } \\
& \text { importjava.util.Random ; } \\
& \text { importjava.util.TimeZone; }
\end{aligned}
$$

For the results of this system:

$$
\begin{aligned}
& \text { importjava.sql.Connection; } \\
& \text { importjava.sql.PreparedStatement; } \\
& \text { importjava.sql.ResultSet; } \\
& \text { importjava.sql.SQLException; } \\
& \text { importjava.util.logging.Level; } \\
& \text { importjava.util.logging.Logger; } \\
& \text { importjavax.swing.JOptionPane; } \\
& \text { importnet.proteanit.sql.DbUtils; }
\end{aligned}
$$

"Fig. 5," shows photo of the main window is designed with all functions that are used by the player and computer/administrator of this system for a new kind of Sudoku game according to the main classes of Java program above.

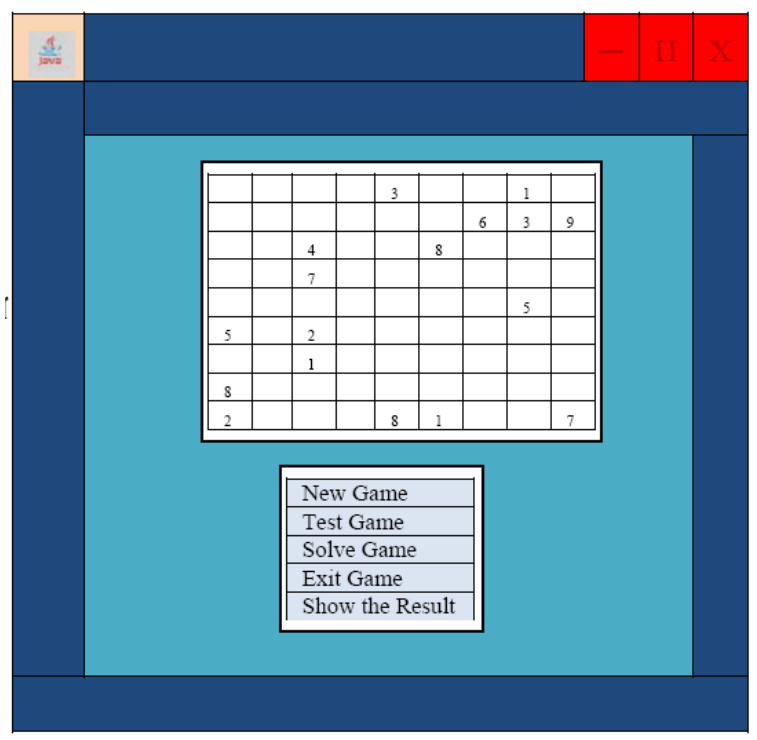

Fig. 5. The main window of (ICSGJS) system.

\section{System's Results}

In addition to the main window that is shown in "Fig. 5" the computer starts calculating the spent time and recode full name for each player plays this game. The computer records all names of players with their spent time by seconds one by one in SQLITE database. After that, It shows their results by the table as shown in Table I below. And then, It will be easy to know which player wins and more intelligent than others by the least time is spent to solve the Sudoku puzzle [8], [9].

TABLE I: ALL PLAYERS WITH THEIR SPENT TIME

\begin{tabular}{|c|c|}
\hline Name of the player & The spent time by seconds \\
\hline First player & $1800 \mathrm{sec}$ \\
\hline Second player & $600 \mathrm{sec}$ \\
\hline $\begin{array}{c}\text { Third player } \\
\text { Until } \\
\text { The last player }\end{array}$ & $1500 \mathrm{sec}$ \\
\end{tabular}

\section{CONCLUSION}

Using engineering design principles and practice an applications of Java language with SQLITE database of Sudoku will be created. The design will be flexible allowing users to choose an appropriate grid size based on time constraints and desired challenge. As well the design will pay close attention to memory usage and try to keep it to a minimum. So, the prototype versions will be created with the final version to be completed and in the future we can focus on advanced topics like Intelligence Competition Functionality and Sudoku Game with Java Software (ICFSGJS) that they make this game more interesting for study and play.

\section{ACKNOWLEDGMENT}

I would like to thank all my partners who work as a programmers by Java language in our universities in Libya "www.misuratau.edu.ly, www.benghaziu.edu.ly" and replied to my endless queries without any complain. I offer my gratitude to my teachers from Malaysia for giving me valuable comments on the contents of this proposal.

\section{REFERENCES}

[1] S. A. Edwards, Sudoku Game Design Language (SGDL), Columbia University, Dec. 20, 2010.

[2] Sudoku. (Jan. 2014). [Online]. Available: http://en.wikipedia.o $\mathrm{rg} /$ wiki/Sudoku

[3] E. Cronin, B. Filstrup, A. R. Kurc, and S. Jamin, A Distributed Multiplayer Game Server System, Electrical Engineering and Computer Science Department University of Michigan, Ann Arbor, MI 48109-2122, May 4, 2001.

[4] Flowchart. (Jan 2014). [Online]. Available: http://en.wikipedia.org/wiki/Flowchart

[5] J. W. Satzinger, R. B. Jackson, and S. D. Burd, Systems Analysis and Design in A Changing World, Missouri State University, RBJ and Associates, University of New Mexico, 2009 Course Technology, Printed in Canada.

[6] Official Java Swing tutorials. (2010). [Online]. Available: http://www.theswingconnection.com

[7] Stackoverflow. (June 2013). [Online]. Available: http://stackoverflow.com/questions/17062055/can-not-connect-to-sqllite

[8] JavaGaming.org-Java technology game development forum. (2010). [Online]. Available: http://www.javagaming.org

[9] Java Forum. (2009). [Online]. Available: http://forum.java.sun.com

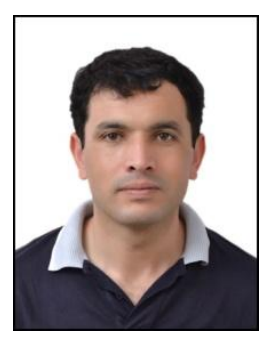

Abdulmonem I. Shennat was born on May 20, 1975 in Misurata city, Libya. He received his bachelor degree of technology in the Department of Electronics Engineering, Communication Division from the Faculty of Industrial technicality in Misurata, Libya from 1999 to 2000 . He obtained his master of science ICT( information communication technology ) from UUM University Utara Malaysia from 2007 to 2008. He had studied courses in Cisco Certified Network Associate (CCNA), Microsoft 
Certified Systems Engineer (MCSE), and Java 2 Enterprise Edition (J2EE) from UUM in Malaysia 2008. And English Language courses until upper Intermediate Level from British Council in Libya in 2009. He worked at the Higher Institute of polytechnic-Misurata, Libya as an engineer in the field of electronics, especially in communication engineering and MATLAB program. During his study in UUM University Utara Malaysia 2007/2008, he worked a little in PHP (hypertext preprocessor) language and WML(Wireless markup language). He was working as a Business\& Contract Manager in Almasarra Company for medical service and LCIC (Libyan company For inspection and conformity) Company in Tripoli/Libya for ISO certification in consultancy department 2009/2010. He was working as a lecturer for some communications and computer courses in Higher Institute of Polytechnic in Misurata/Libya, and Faculty of Electronics Technicality in
Tripoli/Libya 2010. From Jan Feb 2012 until now, he has been working as a lecturer in faculty of IT in Musirata University in Libya www.misurata.edu.ly and his email is ashennat@umit.edu.ly or abdulmonem_ibrahim@hotmail.com. Mr. Shennat is a member of (Federation of Arab Engineers). And he had participated in workshope on model-based system for industrial applications for Prof. Dr. Peter Struss (TechnischeUniversitatMunchen) and participated in IT (information technology) skills awareness day, in UUM. Mr. Shennat was working in maintenance clinics from last March 2011 until Jun 2011 and from Jun 2011 he started to work in MSF organization (Doctors without borders) as a logistic engineer until Jan. 2012. 